\title{
Langevin equation approach to diffusion magnetic resonance imaging
}

\author{
Jennie M. Cooke, ${ }^{1}$ Yuri P. Kalmykov, ${ }^{2}$ William T. Coffey ${ }^{3}$ and Christian M. Kerskens ${ }^{1}$ \\ ${ }^{1}$ Institute of Neuroscience, Trinity College, Dublin 2, Ireland \\ ${ }^{2}$ Laboratoire de Mathématiques, Physique et Systèmes, Université de Perpignan, 52 Avenue de Paul Alduy, \\ 66860 Perpignan Cedex, France \\ ${ }^{3}$ Department of Electronic and Electrical Engineering, Trinity College, Dublin 2, Ireland
}

(Received 29 July 2009; published 2 December 2009)

\begin{abstract}
The normal phase diffusion problem in magnetic resonance imaging (MRI) is treated by means of the Langevin equation for the phase variable using only the properties of the characteristic function of Gaussian random variables. The calculation may be simply extended to anomalous diffusion using a fractional generalization of the Langevin equation proposed by Lutz [E. Lutz, Phys. Rev. E 64, 051106 (2001)] pertaining to the fractional Brownian motion of a free particle coupled to a fractal heat bath. The results compare favorably with diffusion-weighted experiments acquired in human neuronal tissue using a 3 T MRI scanner.

DOI: 10.1103/PhysRevE.80.061102

PACS number(s): 05.40.-a, 87.19.1f, 82.56.Pp, 05.45.Df
\end{abstract}

\section{INTRODUCTION}

During signal acquisition in magnetic resonance imaging (MRI), nuclear magnetic moments are manipulated via a combination of static, gradient, and radiofrequency magnetic fields. These fields and their relative timing (or pulse sequences) can be varied in many ways in order to create image contrast based on characteristics of the medium, tissue, or pathology. In addition to varying the tissue contrast, flowing, diffusing, and perfusing spins can be encoded in the image signal. The clinical applications of diffusion MRI are numerous, and changes in water diffusion in neuronal tissues have been associated with alterations in physiological and pathological states. These include the early detection of acute stroke [1], functional brain imaging [2], white matter fiber tracking [3], the detection of multiple sclerosis [4], and tumors [5].

The precession and relaxation of the net magnetization, as a result of the spin manipulation, is described by the phenomenological Bloch equations [6]. In liquids, however, the positions of the molecules fluctuate due to Brownian motion, so that the Larmor precession is affected, causing dephasing of the resonance signal. In other words, the magnetic field is not constant in space, but has a field gradient $\mathbf{G}$ defining the magnitude of the field at the site of a nucleus given by the position vector $\mathbf{r}$, which is now a stochastic process causing phase fluctuations,

$$
\Delta \Phi(t)=\int_{0}^{t} \omega\left(t^{\prime}\right) d t^{\prime}=\gamma \int_{0}^{t} \mathbf{r}\left(t^{\prime}\right) \cdot \mathbf{G}\left(t^{\prime}\right) d t^{\prime},
$$

where $\gamma$ is the gyromagnetic ratio. Dephasing due to random modulation of the Larmor frequency $\omega(t)$ was first observed by Hahn [7], who noted the attenuation of the observed transient signals in NMR experiments due to the self-diffusion of "spin-containing liquid molecules."

The Bloch equations as originally formulated [6] ignore the Brownian motion of the liquid nuclei. Consequently, numerous attempts to incorporate it have been made $[7,8]$, e.g., that of Carr and Purcell [9]. Their treatment (effectively Einstein's theory $[10,11]$ adapted to the phase fluctuations) assumes that a nucleus in a liquid executes a discrete-time random walk, due to the cumulative effect of very large numbers of impacts of the surrounding particles, so that the phase is a sum of random variables each having arbitrary distributions. The only random variable is the orientation of the walker, i.e., the direction of the jump-length vector [11] as it has finite variance and the waiting time between jumps has finite mean. The problem is always to find the probability that the walker will be in state $n$ at some time $t$ given that it was in a state $m$ at some earlier time, giving rise in general to a difference equation [12-14]. However, by the central limit theorem [11] the dephasing effect may be calculated explicitly in the continuum limit of extremely small mean-square displacements in infinitesimally short times. The above analysis was later much simplified by Torrey [15]. He avoided the problem of explicitly passing to the continuum limit by simply adding (adapting a method of Einstein; see Ref. [11], Chap. 1) a magnetization diffusion term to the transverse magnetization in the Bloch equations, resulting in a partial differential equation, now called the Bloch-Torrey equation $[15,16]$. Moreover, by the introduction of appropriate boundary conditions, this equation is ideally suited to describing restricted diffusion in a confining domain [17]. The Bloch-Torrey equation may be easily solved for nuclei diffusing freely in an infinite reservoir. Thus, Torrey obtained for the dephasing following the application of a step gradient of magnitude $G$ in a liquid characterized by a diffusion coefficient $D$,

$$
\left\langle e^{i \Delta \Phi}\right\rangle=A(t) / A(0)=e^{-D \gamma^{2} G^{2} t^{3} / 3} .
$$

Moreover, for a simple bipolar gradient echo experiment with gradients of strength $G$ and duration $\tau$,

$$
\left\langle e^{i \Delta \Phi}\right\rangle_{G E}=A(2 \tau) / A(0)=e^{-2 D \gamma^{2} G^{2} \tau^{3} / 3} .
$$

The spin-echo diffusion experiment case is slightly different [18] and the calculations are considerably more involved than in the gradient echo one where the second gradient pulse has the effect of resetting the dephasing caused by the first pulse. By applying the $180^{\circ}$ pulse in the spin-echo experiment, the phase is reset by double the extent to which it was advanced [18], so that 


$$
\left\langle e^{i \Delta \Phi}\right\rangle_{S E}=e^{-D \gamma^{2} G^{2} \delta^{2}(\Delta-\delta / 3)}=e^{-b D} .
$$

In Eq. (4) $\delta$ is the gradient spacing and $\Delta$ is the time interval from the starting time of the first gradient to the starting time of the rephasing gradient. The notation $b=\gamma^{2} G^{2} \delta^{2}(\Delta-\delta / 3)$ is traditionally used as in Eq. (4) for the simplification and the degree of diffusion weighting in an MRI acquisition is set using a $b$ value. The diffusion weighting (or $b$-value) is increased, typically by increasing the strength of the diffusionweighting gradient $G$.

Now Eqs. (2)-(4) describe precisely the signal loss due to the translational motion of the magnetic moments in unrestricted (free) water in a magnetic resonance experiment. However, difficulties arise when these equations are applied in vivo because these simple single exponential equations fail to describe the diffusion of water in tissue. An empirical approach to this problem has been to assume that fast and slow diffusion components exist [19], so that the decay may be described by the simple equation

$$
A(t) / A(0)=V_{1} e^{-b D_{1}}+V_{2} e^{-b D_{2}} .
$$

Here, $V_{1}$ and $V_{2}$ are considered as the volume fractions of protons in intracellular and extracellular spaces in neuronal structures with the distinct diffusion coefficients $D_{1}$ and $D_{2}$. This equation, which is useful in practice [20], has a simple theoretical explanation, namely, the signal from two separate compartments is the sum of the signals from each compartment although this statement should be regarded as approximate because of the presence of a boundary. Yet another approach is that of Bennett et al. [21] who used the stretched-exponential expression or Kohlrausch-WilliamsWatts form [11]

$$
A(t) / A(0)=e^{-\left(b D_{D C}\right)^{\alpha}},
$$

where $\alpha \neq 1$ is the stretch parameter and $D_{D C}$ is the distributed diffusion coefficient arising from fitting Eq. (6) to data. The parameter $\alpha$ could be used to measure the heterogeneity of the dephasing process in tissue, which appears to exhibit anomalous diffusion behavior, signified by a mean-square displacement of the form $t^{\alpha}$. In general the motion can be either subdiffusive $(\alpha<1)$, which signifies a slow relaxation process, or superdiffusive $(\alpha>1)$, which leads to turbulence. As far as one possible explanation of Eq. (6) based on the microscopic origins of anomalous diffusion is concerned, we remark that the finite jump-length variance and the finite average jump time, in the theory of the normal Brownian motion, define a physical length scale and a physical time scale $[22,23]$ so that the central limit theorem applies. In anomalous diffusion, however, either the second moment of the jump-length distribution or the first moment of the jump time distribution diverges or indeed both of them. Such motions are invariably characterized by heavy tailed probability distributions (i.e., power-law tails) so that the central limit theorem no longer applies [24,25]. They are known by the generic title of continuous-time random walks (CTRWs) [26]. Examples are the Lévy stable motion for which the mean-square displacement diverges due to the occurrence of very long jumps [27], and the specialized CTRW with a long-tailed waiting time probability distribution where the walker may remain in a given configuration for an arbitrarily long period before undertaking a jump of finite mean-square length, so that the mean waiting time diverges. Here, $\alpha$ is $[22,23]$ the fractal dimension of the set of waiting times, which is the scaling of the waiting time segments in the random walk with magnification. Hence, it is impossible to attribute underlying physical scales to such processes. For example, the origin of the divergent first moment of the jump times may be due to a random distribution of impurities within a matrix, so that a spatial disorder corresponding to an energetic disorder will give rise to a temporal disorder resulting in anomalous diffusion. Yet another microscopic explanation (the one which will be used in this paper) is that the anomalous behavior simply arises from the inclusion of memory effects [11] in the normal Brownian motion, so destroying its Markovian character.

The use of anomalous diffusion to describe relaxation behavior is very well established in many fields of physics, including biophysics and physics in medicine $[23,27,28]$. In the particular case of subdiffusive transport, for example, we mention such diverse phenomena as charge carrier transport in amorphous semiconductors, diffusion in percolative or porous systems, transport in fractal geometries, as well as protein conformational dynamics [24,29-33]. In the NMR context the anomalous diffusion approach was expanded upon by Magin et al. [34], where the Bloch-Torrey equation was converted to fractional form.

However, in using random-walk models in the context of microscopic explanations, it should be noted that diffusion in tissue is restricted or hindered; that is, it does not take place in an infinite reservoir and so may lead to very different signal attenuations [17]. For instance, Robertson [35] described the motional narrowing long-time regime for diffusion between parallel planes when the signal decays exponentially in time, unlike $t^{3}$ as it is for unrestricted diffusion. Stejskal and Tanner [36] showed that the signal has oscillatory behavior for narrow gradient pulses and the related diffusion-diffraction patterns were observed by Callaghan et al. [37]. The localization regime predicted by Stoller et al. [38] exhibits a stretched-exponential behavior. In these and many other cases $[17,39]$, diffusion may be considered as normal, and it is a geometrical restriction alone that may lead to deviations from the classical unrestricted diffusion.

Hitherto virtually all the microscopic approaches to anomalous diffusion in the context of resonant imaging ultimately rely on the probability distribution of the phase, a notable exception being that of Widom and Chen [32] who used a frequency domain analysis based on the spectral function characterizing fractal Brownian motion. However, since the underlying stochastic process is the position of a nucleus, it appears that a much more transparent treatment of the phase diffusion could be achieved by means of the Langevin equation. For normal diffusion, this is simply the Newtonian equation of motion of the nucleus, augmented by a systematic frictional force proportional to the velocity, superimposed on which is a very rapidly fluctuating random force, both representing the effect of the surrounding heat bath on the nucleus. Here, we outline how the normal phase diffusion problem may be treated by means of the Langevin equation using simply the properties of the characteristic function of 
Gaussian random variables. Moreover, in order to provide a possible theoretical explanation for the empirical equation (6), in terms of the memory effects alluded to above, we shall show how that treatment may be simply extended to anomalous diffusion using a fractional generalization of the Langevin equation proposed by Lutz [33]. This equation pertains to the fractional Brownian motion of a free particle coupled to a fractal heat bath, and so [33] unlike the CTRW it describes Gaussian transport with the non-Markovian character being expressed via a memory function. The results will then be compared with diffusion-weighted experiments, which were acquired in human brain tissue using a $3 \mathrm{~T}$ MRI scanner.

\section{PHASE DIFFUSION DUE TO THERMAL AGITATION}

The starting point of our treatment of dephasing is the work of Bloch [6]. He proposed in his phenomenological treatment of nuclear induction the differential equation for the time dependence of the macroscopic nuclear magnetization $\mathbf{M}(t)$ under the influence of an external field $\mathbf{H}(t)$, viz.,

$$
\frac{d \mathbf{M}}{d t}=\gamma \mathbf{M} \times \mathbf{H}-\mathbf{i} \frac{M_{x}}{T_{2}}-\mathbf{j} \frac{M_{y}}{T_{2}}-\mathbf{k} \frac{M_{z}-M_{0}}{T_{1}},
$$

where $\gamma=\mu / I \hbar$ is the gyromagnetic ratio of the nuclei under consideration with magnetic moment $\mu$ and spin $I$ and $\mathbf{i}, \mathbf{j}$, and $\mathbf{k}$ are the usual triad of unit vectors along the Cartesian axes. The external field has the form

$$
\mathbf{H}(t)=\mathbf{k} H_{0}+\mathbf{H}_{1}(t),
$$

where $H_{0}$ is strong and constant while $\mathbf{H}_{1}$ is relatively weak and an arbitrary function of time. $M_{0}$ is the equilibrium magnetization in the field $H_{0}$ and the establishment of thermal equilibrium is in Eq. (7) described by two relaxation time constants $T_{1}$ and $T_{2}$ - the longitudinal and transverse relaxation times, respectively-meaning that in the absence of the transverse field $H_{1}$ the $x$ and $y$ components will vanish with a time constant $T_{2}$, while the equilibrium magnetization will be attained with a time constant $T_{1}$. To study relaxation we suppose that $H_{1}$ is zero while $H_{0}$ is slightly altered in order to induce relaxation; then the Bloch equations become

$$
\frac{d}{d t} \mathbf{M}=\gamma\left(\mathbf{i} M_{y} H_{0}-\mathbf{j} M_{x} H_{0}\right)-\mathbf{i} \frac{M_{x}}{T_{2}}-\mathbf{j} \frac{M_{y}}{T_{2}}-\mathbf{k} \frac{M_{z}-M_{0}}{T_{1}} .
$$

Clearly the transverse $\left(M_{x}, M_{y}\right)$ and the longitudinal $M_{z}$ components of $\mathbf{M}$ decouple in the absence of $H_{1}$ and forming the complex variable $M_{\perp}(t)=M_{x}(t)+i M_{y}(t)$ we then have

$$
\dot{M}_{\perp}=-i \gamma M_{\perp} H_{0}-M_{\perp} / T_{2} .
$$

The solution of this differential equation following perturbation of the constant field $H_{0}$ is simply

$$
M_{\perp}(t)=M_{\perp}(0) e^{-\left(i \omega_{0}+1 / T_{2}\right) t},
$$

where $\omega_{0}=\gamma H_{0}$ is the Larmor precessional frequency. Equation (11) represents a decaying oscillation. In practice $H_{0}$ is not constant in space and so it has a field gradient defining the magnitude of the field at the site of a nucleus given by the position vector $\mathbf{r}(t)$,

$$
H(\mathbf{r}, t)=\mathbf{r}(t) \cdot \nabla H(z, t)=\mathbf{r}(t) \cdot \mathbf{G}(z, t),
$$

so that solution (11) alters to

$$
M_{\perp}(\mathbf{r}, t)=M_{\perp}(\mathbf{r}, 0) \exp \left[-t / T_{2}-i \gamma \int_{0}^{t} \mathbf{r}\left(t^{\prime}\right) \cdot \mathbf{G}\left(z, t^{\prime}\right) d t^{\prime}\right] .
$$

Hence, the transverse magnetization is now a function of the position of the nucleus. However, Eq. (13) omits the Brownian motion of the particles in the liquid, which carry the nuclei. This must be taken account of in resonant imaging. Thus, $\mathbf{r}$ is now a stochastic process. Hence, Eq. (10) becomes the stochastic differential equation,

$$
\dot{M}_{\perp}=-\left(i \gamma \mathbf{r} \cdot \mathbf{G}+1 / T_{2}\right) M_{\perp} .
$$

Equation (14) now represents the Langevin equation of the process [11]. We can simplify the problem, so that the damping term is incorporated into the time derivative, and simply calculate the dephasing effect. Hence, we see that the dephasing due to the thermal motion of the nuclei bearing the magnetic moments is obtained by calculating the mean value of the functional $\left\langle e^{i \Delta \Phi}\right\rangle$, where

$$
\Delta \Phi=-\gamma \int_{0}^{t} \dot{\mathbf{r}}\left(t_{1}\right) \int_{0}^{t_{1}} \mathbf{G}\left(t^{\prime}\right) d t^{\prime} d t_{1}
$$

is the dephasing. Equation (15) is obtained by integration by parts from Eq. (1) by imposing the so-called "rephasing condition" $\int_{0}^{t} \mathbf{G}\left(t^{\prime}\right) d t^{\prime}=0$. Clearly, the calculation of $\left\langle e^{i \Delta \Phi}\right\rangle$ merely amounts to determining the characteristic function of the centered random variable $\Delta \Phi$. This is particularly easy for centered Gaussian processes because then one may write [6]

$$
\left\langle e^{i \Delta \Phi}\right\rangle=e^{-\left\langle\Delta \Phi^{2}\right\rangle / 2} .
$$

Thus, if we regard the particles carrying the nuclei as free Brownian particles, we can determine the dephasing by means of Eq. (16). It should be noted that the treatment proposed here differs from that of Torrey [15] and Magin et al. $[34(\mathrm{a})]$ although ultimately leading to the same result as they started by writing down Eq. (10) and, following Einstein [10], added a term $D \nabla^{2} M_{\perp}$ to account for the average dephasing, where $D$ is the diffusion coefficient. It follows that $D$ can then be measured via the amplitude of the echo signal from nuclear spins subject to an appropriate sequence of magnetic field pulses.

\section{NONINERTIAL DIFFUSION}

In order to illustrate the calculation of the dephasing from the Langevin equation we consider for simplicity the Brownian motion of a free particle along the $x$ axis. We shall first derive Eq. (2) for the phase diffusion, which corresponds to the noninertial limit, where the inertia of the particle may be ignored. Here, the Langevin equation is simply [11] 


$$
\zeta \dot{X}(t)=\lambda(t) .
$$

where $X(t)$ is the coordinate of the Brownian particle; $\lambda(t)$ is a random force with white noise properties

$$
\langle\lambda(t)\rangle=0, \quad\left\langle\lambda\left(t_{1}\right) \lambda\left(t_{2}\right)\right\rangle=2 \zeta k T \delta\left(t_{1}-t_{2}\right),
$$

with the angular brackets denoting the average over the realizations of $\lambda(t)$; $\zeta$ is the viscous drag coefficient imposed on a translating molecule by its surroundings and Stokes' law is assumed to apply; $\zeta \dot{X}(t)$ represents the average retarding effect of the heat bath and the noise, $\lambda(t)$, the extremely rapid fluctuations about this average value due to molecular collisions; $\delta(t)$ is the Dirac delta function; $k$ is Boltzmann's constant; and $T$ is the absolute temperature. Equation (17) follows from the inertial Langevin equation for the velocity $v(t)=\dot{X}(t)$ of the Brownian particle of mass $m$, viz.,

$$
m \dot{v}(t)+\zeta v(t)=\lambda(t),
$$

by neglecting the inertial term.

According to Eq. (15) the noninertial Langevin equation for the phase $\Phi(t)$ is

$$
\dot{\Phi}(t)=-\gamma \dot{X}(t) \int_{0}^{t} G\left(t^{\prime}\right) d t^{\prime}=-\gamma \zeta^{-1} \lambda(t) \int_{0}^{t} G\left(t^{\prime}\right) d t^{\prime} .
$$

These equations simply express the fact that the only way the phase can change is via the equation of motion of $X(t)$. In the Brownian motion of a free particle, the phase $\Phi(t)$ is a centered Gaussian random variable with variance $\sigma^{2}=\left\langle\Delta \Phi^{2}\right\rangle$ $=\left\langle\Phi^{2}\right\rangle$ since $\langle\Phi\rangle=0$ and $t_{0}=0$. Noting that

$$
\Phi^{2}(t)=2 \int_{0}^{t} \Phi\left(t_{1}\right) \dot{\Phi}\left(t_{1}\right) d t_{1},
$$

because we may take $\Phi(0)=0$, we have for a step field gradient

$$
\begin{aligned}
\left\langle\Delta \Phi^{2}\right\rangle(t)= & 2 \gamma^{2} \zeta^{-2} \int_{0}^{t} \int_{0}^{t_{1}} \int_{0}^{t_{1}} G\left(t^{\prime}\right) d t^{\prime} \\
& \times \int_{0}^{t_{2}} G\left(t^{\prime \prime}\right) d t^{\prime \prime}\left\langle\lambda\left(t_{1}\right) \lambda\left(t_{2}\right)\right\rangle d t_{1} d t_{2} \\
= & 2 D \gamma^{2} \int_{0}^{t}\left[\int_{0}^{t_{1}} G\left(t^{\prime \prime}\right) d t^{\prime \prime}\right]^{2} d t_{1} \\
= & \frac{2}{3} D \gamma^{2} G^{2} t^{3}
\end{aligned}
$$

where $D=k T / \zeta$ is the diffusion coefficient, which is defined via the mean-square displacement $\left\langle[X(t)-X(0)]^{2}\right\rangle=2 D t$ of the Brownian particle in a time interval $t$. Hence, from Eq. (16) we have the known result (2) for the dephasing [9] following the application of a step gradient.

The gradient echo result (3) may be obtained in the same manner. For the spin-echo case Eq. (4) may be obtained by writing the left-hand side of Eq. (21) in the notation of Stejskal and Tanner [18]

$$
\left\langle\Delta \Phi^{2}\right\rangle(2 \tau)=2 D \gamma^{2} \int_{0}^{2 \tau}\left[F^{2}\left(t_{1}\right)+2(\xi-1) \mathbf{f} \cdot \mathbf{F}\left(t_{1}\right)+f^{2}\right] d t_{1}
$$

with $\xi=+1$ for $t<\tau$ and $\xi=-1$ for $t>\tau$, where $\mathbf{F}(t)$ is defined by

$$
\mathbf{F}(t)=\int_{0}^{t} \mathbf{G}\left(t^{\prime}\right) d t^{\prime}
$$

and $\mathbf{f}=\mathbf{F}(\tau)$, where $\tau$ is the time of application of the $180^{\circ}$ pulse.

\section{PHASE DIFFUSION INCLUDING THE INERTIA}

The analysis given above ignores the inertia of the Brownian particles. If the inertial effects are included the translational process, $X(t)$ now possesses two characteristic times. One characterizes the slow diffusion associated with the noninertial motion, which we have already analyzed. The other is the correlation time $\tau_{v}=m / \zeta$ of the velocity correlation function. It is of interest to include these in the phase diffusion and therefore we show how the calculation just outlined using the noninertial Langevin equation [Eq. (17)] may be extended for a free particle of mass $m$. In the inertial motion of a Brownian particle described by Eq. (18), an explicit formula for the displacement $X(t)$ is available from the Ornstein-Uhlenbeck theory $[11,40]$. We have from Eq. (18), where without loss of generality we may set $x_{0}=0$ when $t_{0}=0$,

$$
\begin{gathered}
\dot{X}(t)=v_{0} e^{-\beta t}+\frac{1}{m} \int_{0}^{t} e^{-\beta\left(t-t^{\prime}\right)} \lambda\left(t^{\prime}\right) d t^{\prime}, \\
X(t)=\frac{v_{0}}{\beta}\left(1-e^{-\beta t}\right)+\frac{1}{m \beta} \int_{0}^{t}\left(1-e^{-\beta\left(t-t^{\prime}\right)}\right) \lambda\left(t^{\prime}\right) d t^{\prime},
\end{gathered}
$$

where $\beta=\zeta / m$. If we now assume a Maxwellian distribution of velocities for the initial velocity $v_{0}$, we have [11] from Eqs. (23) and (24) the Ornstein-Uhlenbeck result [40] for the mean-square displacement of a Brownian particle including the inertia

$$
\left\langle X^{2}(t)\right\rangle=\frac{2 k T}{\beta^{2} m}\left(\beta t-1+e^{-\beta t}\right),
$$

as well as the velocity correlation function

$$
\left\langle\dot{X}\left(t_{1}\right) \dot{X}\left(t_{2}\right)\right\rangle=(k T / m) e^{-\beta\left|t_{1}-t_{2}\right|} .
$$

Now for a step field gradient we again have

$$
\dot{\Phi}(t)=-\gamma G t \dot{X}(t), \quad \Phi(t)=-\gamma G \int_{0}^{t} t_{1} \dot{X}\left(t_{1}\right) d t_{1} .
$$

Hence, using Eqs. (20) and (26), we can evaluate the meansquare value of the phase $\left\langle\Delta \Phi^{2}\right\rangle(t)$ as 


$$
\begin{aligned}
\left\langle\Delta \Phi^{2}\right\rangle(t) & =2 \gamma^{2} G^{2} \int_{0}^{t} \int_{0}^{t_{1}} t_{1} t_{2}\left\langle\dot{X}\left(t_{1}\right) \dot{X}\left(t_{2}\right)\right\rangle d t_{1} d t_{2} \\
& =\frac{\gamma^{2} G^{2} k T}{3 \beta^{4} m}\left[6+t^{2} \beta^{2}(2 t \beta-3)-6 e^{-\beta t}(1+t \beta)\right],
\end{aligned}
$$

which reduces to the Carr-Purcell-Torrey result [Eq. (21)] for long times $t \beta \gg 1$. For short times, $t \beta \ll 1$, we have the purely kinematic result

$$
\left\langle\Delta \Phi^{2}\right\rangle(t) \approx \frac{\gamma^{2} G^{2} k T}{4 m} t^{4} .
$$

Again $\Delta \Phi$ is a linear transformation of a Gaussian random variable, so that by the properties of characteristic functions

$$
\left\langle e^{i \Delta \Phi}\right\rangle=e^{-\left\langle\Delta \Phi^{2}\right\rangle / 2} \text {. }
$$

Hence, Eq. (27) yields the inertia corrected dephasing for a step gradient. In general an infinity of fast relaxation modes will be generated due to the double transcendental nature of this function and one dominant much slower mode, which is that associated with the slow diffusive motion [c.f. Eq. (2)]. An obvious generalization of the left-hand side of Eq. (27) for arbitrary gradient shapes defined by

$$
\mathbf{F}(t)=\int \mathbf{G}\left(t^{\prime}\right) d t^{\prime}
$$

is

$$
\left\langle\Delta \Phi^{2}\right\rangle(t)=2 \gamma^{2} \int_{t} \int_{t_{1}}\left\langle\dot{X}\left(t_{1}\right) \dot{X}\left(t_{2}\right)\right\rangle F\left(t_{1}\right) F\left(t_{2}\right) d t_{1} d t_{2} .
$$

Hence, in order to calculate the dephasing for a Gaussian process all that is required is a knowledge of the velocity correlation function and the precise form of the field gradients. We remark that Eq. (30) was previously derived by Stepišnik and Callaghan [41-43] in connection with measurement of flow by NMR spectroscopy and long-time tails of the molecular velocity correlation function in a confined fluid.

\section{FRACTIONAL DIFFUSION}

Here, our objective is to provide one of many possible microscopic explanations for the empirical stretched exponential (6) of Bennett et al. [21]. Our particular hypothesis is that it may be explained via memory effects giving rise to fractional Brownian motion (which preserves a few of the features of the CTRW) and its associated Langevin equation. Thus, we note that Lutz [33] introduced the following fractional Langevin equation for the translational motion of a free Brownian particle:

$$
m \frac{d}{d t} v(t)+m \beta_{\alpha 0} D_{t}^{\alpha-1} v(t)=\lambda(t),
$$

where $\beta_{\alpha}$ is the friction coefficient and $m \beta_{\alpha{ }_{0}} D_{t}^{\alpha-1} v(t)$ and $\lambda(t)$ are, respectively, the generalized frictional and random forces with the properties

$$
\langle\lambda(t)\rangle=0, \quad\left\langle\lambda\left(t^{\prime}\right) \lambda(t)\right\rangle=\frac{m k T \beta_{\alpha}}{\Gamma(1-\alpha)}\left|t-t^{\prime}\right|^{-\alpha}
$$

( $\Gamma$ denotes the gamma function). The Riemann-Liouville fractional derivative is defined by [44]

$$
{ }_{0} D_{t}^{-\sigma} g(t)=\frac{1}{\Gamma(\sigma)} \int_{0}^{t} \frac{g\left(t^{\prime}\right)}{\left(t-t^{\prime}\right)^{1-\sigma}} d t^{\prime}, \quad 0<\sigma<1
$$

and has the form of a memory function, so that Eq. (31) may be regarded as a generalized Langevin equation [11,33]

$$
m \frac{d}{d t} v(t)+\int_{0}^{t} K_{\alpha}\left(t-t^{\prime}\right) v\left(t^{\prime}\right) d t^{\prime}=\lambda(t) .
$$

The memory function $K_{\alpha}(t)$ is given (in accordance with the fluctuation dissipation theorem) by the autocorrelation function $K_{\alpha}(t)=\langle\lambda(0) \lambda(t)\rangle /(k T)$. Lutz also supposed that the random force $\lambda(t)$ is Gaussian; thus, Eq. (31), which describes Gaussian transport, is capable of reproducing the stretchedexponential behavior associated with anomalous diffusion and has the merit unlike possible CTRW treatments that Eq. (16) still applies, i.e., a knowledge of the first two moments is sufficient to calculate all the higher-order moments and so the characteristic function. Nevertheless, the process described by the Langevin equation has a mean-square displacement which is the same as that ensuing from the CTRW and reproduces some of its features however failing to reproduce many others such as weak ergodicity breaking [29]. We remark that the fractional Langevin equation (31) may also be used to study both subdiffusion and superdiffusion of a particle coupled to a fractal heat bath [33].

The formal exact solution of Eq. (31) may be obtained using Laplace transforms [33] [c.f. Eq. (11.76) of [11]]; we have

$$
\begin{aligned}
\dot{X}(t)= & \dot{X}(0) E_{2-\alpha, 1}\left(-t^{2-\alpha} \beta_{\alpha}\right)+m^{-1} \int_{0}^{t} \lambda\left(t^{\prime}\right) \\
& \times E_{2-\alpha, 1}\left[-\left(t-t^{\prime}\right)^{2-\alpha} \beta_{\alpha}\right] d t^{\prime}
\end{aligned}
$$

and

$$
\begin{aligned}
X(t)= & X(0)+\int_{0}^{t} \dot{X}\left(t^{\prime}\right) d t^{\prime} \\
= & X(0)+\dot{X}(0) t E_{2-\alpha, 2}\left(-\beta_{\alpha} t^{2-\alpha}\right)+m^{-1} \int_{0}^{t} \int_{0}^{t^{\prime \prime}} \lambda\left(t^{\prime}\right) \\
& \times E_{2-\alpha, 1}\left[-\left(t-t^{\prime}\right)^{2-\alpha} \beta_{\alpha}\right] d t^{\prime} d t^{\prime \prime}
\end{aligned}
$$

where $E_{\alpha, \beta}(z)$ is the generalized Mittag-Leffler function defined by $[11,44-46]$

$$
E_{\alpha, \beta}(z)=\sum_{k=0}^{\infty} \frac{z^{k}}{\Gamma(\beta+k \alpha)}, \quad \alpha, \beta>0 .
$$

Here, the Mittag-Leffler function $E_{\alpha}(z)$ is $E_{\alpha, 1}(z)$; furthermore, 


$$
\int_{0}^{t} E_{2-\alpha, 1}\left(-\beta_{\alpha} t^{\prime 2-\alpha}\right) d t^{\prime}=t E_{2-\alpha, 2}\left(-\beta_{\alpha} t^{2-\alpha}\right) .
$$

Thus, we have from Eq. (33) the velocity correlation function for fractional Brownian motion

$$
\langle\dot{X}(0) \dot{X}(t)\rangle=(k T / m) E_{2-\alpha}\left(-\beta_{\alpha} t^{2-\alpha}\right),
$$

where the Mittag-Leffler function $E_{\alpha}$ interpolates between the initial stretched-exponential form

$$
E_{2-\alpha}\left(-\beta_{\alpha} t^{2-\alpha}\right) \sim \exp \left[-\beta_{\alpha} t^{2-\alpha} / \Gamma(3-\alpha)\right]
$$

and the long-time inverse power-law behavior $\left[\beta_{\alpha} t^{2-\alpha} \Gamma(\alpha-1)\right]^{-1}$. Thus, the angular velocity correlation function has initial stretched-exponential behavior accompanied by a slowly decaying long-time tail representing a memory of the initial conditions. Furthermore, noting that $X^{2}(t)$ is given by

$$
X^{2}(t)=2 \int_{0}^{t} X\left(t^{\prime}\right) \dot{X}\left(t^{\prime}\right) d t^{\prime},
$$

[for $X(0)=0]$, we have [33]

$$
\begin{aligned}
\left\langle X^{2}(t)\right\rangle & =\frac{2 k T}{m} \int_{0}^{t} \int_{0}^{t^{\prime}} E_{2-\alpha}\left[-\beta_{\alpha}\left(t^{\prime}-t^{\prime \prime}\right)^{2-\alpha}\right] d t^{\prime \prime} d t^{\prime} \\
& =\frac{2 k T}{m} t^{2} E_{2-\alpha, 3}\left(-\beta_{\alpha} t^{2-\alpha}\right) \sim \frac{2 k T}{m \beta_{\alpha}} \frac{t^{\alpha}}{\Gamma(1+\alpha)}
\end{aligned}
$$

in the long-time limit $\beta t \gg 1$, because for large $z$ the generalized Mittag-Leffler function $E_{a, b}(z)$ has the inverse powerlaw behavior $E_{a, b}(-z) \sim[z \Gamma(b-a)]^{-1}[33]$. According to the definition, the range $0<\alpha<1$ corresponds to subdiffusion in configuration space signifying a slow relaxation process. We remark that Eq. (34) for the velocity correlation function for fractional Brownian motion is identical to the velocity correlation function rendered by the diffusion limit of the CTRW with a power-law distribution of waiting times [11]. Lutz [33] also showed that these completely different forms of non-Markovian anomalous diffusion share a few common characteristics. In particular, they satisfy the same generalized Einstein relation and their lowest moments are all equal with the exception of the second moment of the velocity [11].

Again proceeding for a step field gradient and noting Eq. (27), we have the mean-square phase,

$$
\begin{aligned}
\left\langle\Delta \Phi^{2}\right\rangle(t) & =2 \gamma^{2} G^{2} \int_{0}^{t} \int_{0}^{t_{1}} t_{1} t_{2}\left\langle\dot{X}\left(t_{1}\right) \dot{X}\left(t_{2}\right)\right\rangle d t_{1} d t_{2} \\
& =\frac{2 \gamma^{2} G^{2} k T}{m} \int_{0}^{t} \int_{0}^{t_{1}} t_{1} t_{2} E_{2-\alpha}\left[-\beta_{\alpha}\left(t_{1}-t_{2}\right)^{2-\alpha}\right] d t_{1} d t_{2} .
\end{aligned}
$$

In the noninertial limit, i.e., at long times, $t \beta \gg 1$, again noting the asymptotic behavior of the Mittag-Leffler function at long times, we have

$$
\left\langle\Delta \Phi^{2}\right\rangle(t) \sim \frac{\gamma^{2} G^{2} k T}{m \beta_{\alpha}} \frac{2(1+\alpha)}{\Gamma(3+\alpha)} t^{2+\alpha} .
$$

In the normal diffusion limit, $\alpha \rightarrow 1$, Eq. (38) reduces to Eq. (21). For short times, $t \beta \ll 1$, Eq. (37) yields

$$
\left\langle\Delta \Phi^{2}\right\rangle(t) \approx \frac{\gamma^{2} G^{2} k T}{4 m} t^{4}\left[1-\frac{8 \beta_{\alpha} t^{2-\alpha}}{(6-\alpha) \Gamma(5-\alpha)}+\cdots\right] .
$$

The leading term of expansion (39) coincides with the purely kinematic result (28). Moreover, the gradient echo dephasing (cf. Eqs. (2) and (3)) is simply the r.h.s of Eq. (38).

The spin-echo dephasing is calculated in a similar manner. However, a step function must be introduced to allow for the gap between the gradient pulses. Rather than rectangular gradients, $F\left(t_{1}\right)$ and $F\left(t_{2}\right)$ now represent arbitrary gradient shapes, $t_{1}$ and $t_{2}$ are arbitrary intervals of integration, and for convenience we define $t_{2}>t_{1}$. For the spin-echo case, these functions are defined by

$$
\begin{gathered}
F\left(t_{1}\right) \rightarrow F\left(t_{1}\right) \theta\left(t_{1}\right)-2 \theta\left(t_{1}-\tau\right) F(\tau), \\
F\left(t_{2}\right) \rightarrow F\left(t_{2}\right) \theta\left(t_{2}\right)-2 \theta\left(t_{2}-\tau\right) F(\tau)
\end{gathered}
$$

( $\theta$ representing the unit step function) so that

$$
\left\langle\Delta \Phi^{2}\right\rangle(t)=\frac{2 k T \gamma^{2}}{\beta_{\alpha} m \Gamma(\alpha-1)} \int_{0}^{t} d t_{2} \int_{0}^{t_{2}} \frac{F\left(t_{1}\right) F\left(t_{2}\right) d t_{1}}{\left(t_{2}-t_{1}\right)^{2-\alpha}} .
$$

This expression is evaluated over all regions of integration for the spin-echo sequence (Fig. 1), which employs two positive diffusion gradients of duration $\delta$ around the $180^{\circ} \mathrm{rf}$ pulse (at $t=\tau$ ), where the second gradient begins at $t=\Delta$. We obtain,

$$
\left\langle\Delta \Phi^{2}\right\rangle=\frac{2 \gamma^{2} k T}{m \beta_{\alpha}} \frac{G^{2} \delta^{2}}{\Gamma(1+\alpha)}\left[\frac{2 \delta^{\alpha}}{2+\alpha}+(\Delta-\delta)^{\alpha}\right] .
$$

(See Appendix B for details). In the normal diffusion limit $(\alpha=1)$ this reduces to the Stejskal-Tanner equation (4).

The fractional Brownian motion we have just discussed assumes that the driving force $\lambda(t)$ is Gaussian so that the characteristic function (16) still applies as in the normal Brownian motion. Hence, the decay of the phase remains (albeit stretched) exponential. Thus, the phase as calculated from the fractional Brownian motion unlike that resulting from the diffusion limit of the CTRW, with a power-law distribution of waiting times, does not exhibit the characteristic long-time tail often associated with anomalous diffusion processes, signifying that the diffusion process depends strongly on the initial conditions. If this phenomenon is included it appears no longer possible to treat the dephasing process using the Langevin equation and a fractional diffusion equation similar to that treated in Refs. $[25,30,33,46]$ adapted to the phase must be used, rendering the solution much more complicated.

\section{COMPARISON WITH EXPERIMENT}

The aim of the experimental component of this work was to demonstrate the fractional nature of the diffusion of water 

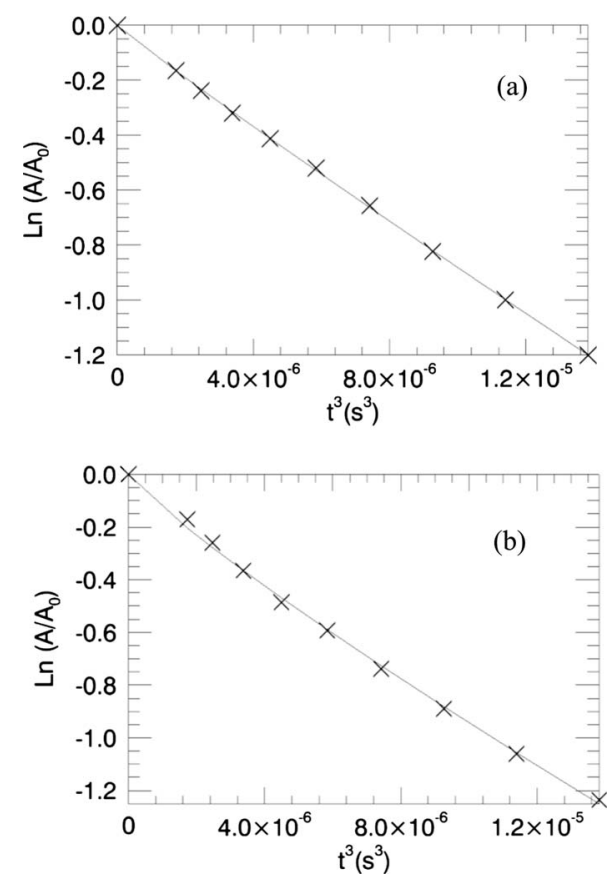

FIG. 1. Plots of data (symbol) and fit (solid line) for (a) gray matter and (b) white matter regions of interest for subject 1 . The image intensity was normalized by dividing by the $b_{0}$ image. The fractional diffusion model [Eq. (44), solid line] was fitted to the experimental data to determine $D$ and $\alpha$ (Tables I and II). The experimental data are plotted against $t^{3}$.

in tissue using MRI. To this end the customized pulse sequence used an increasing duration of the diffusion gradients to increase the diffusion weighting, instead of the more usual increase in the field gradient strength, in a similar approach to that of Latour et al. [47] when the time dependence of water diffusion was investigated. Image data were collected with a 3 T Philips Achieva clinical MRI system, with a gradient coil system which could produce linear field gradients up to $50 \mathrm{mT} / \mathrm{m}$. The rf coil employed was a Philips SENSE head $\mathrm{rf}$ transceiver coil. Diffusion-weighted experiments were performed on nine healthy volunteers: five males and four females. They ranged in age from 19 to 29 , with a mean age of 27 .

The first experiment employed a customized diffusionweighted gradient echo multishot echo-planning imagining (EPI) sequence, which was carried out on each subject in order to compare Eq. (38) with experiment. The strength of the trapezoidal diffusion-weighting gradients was set to 48 $\mathrm{mT} / \mathrm{m}$ and the duration ranged from 12 to $24 \mathrm{~ms}$ in increments of $1.5 \mathrm{~ms}$. The gradients were applied in the axial direction only. The second experiment also used a customized gradient echo EPI sequence; however, the bipolar diffusion-weighting gradients now had a fixed duration of 24 $\mathrm{ms}$ and the gradient strength was varied over eights steps from 8 to $48 \mathrm{mT} / \mathrm{m}$. For both experiments this would equate to a maximum $b$ value of $1460 \mathrm{~s} / \mathrm{mm}^{2}$ as defined in Eq. (2). An image with no diffusion weighting (a $b_{0}$ image) was acquired at the start of each weighting sequence. A sensitivity encoding a factor of 2 was applied. The repetition time was 2 $\mathrm{s}$ and the echo time was $64.2 \mathrm{~ms}$. Ten $4 \mathrm{~mm}$ axial slices with
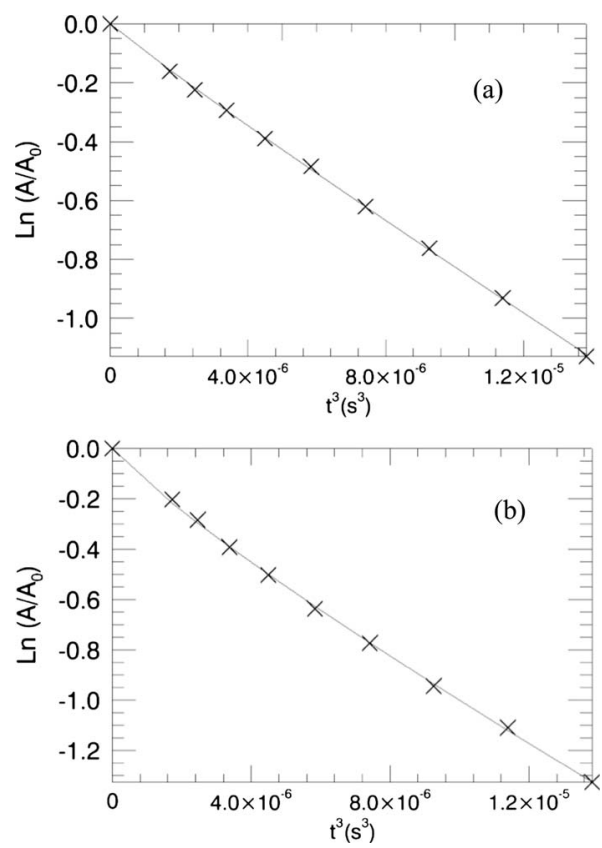

FIG. 2. Plots of data (symbol) and fit (solid line) for (a) gray matter and (b) white matter regions of interest for subject 2 .

a field of view of $23 \times 23 \mathrm{~cm}^{2}$ were acquired to encompass the whole brain. The ten diffusion weightings were repeated sequentially a total of 30 times for each slice for averaging purposes. Inversion recovery images of each subject were also acquired as a map of gray and white matters.

Postprocessing and fitting were performed using IDL 7.0 (ITT Visual Information Solutions, Boulder, CO, USA). The experiments were averaged over the 30 repetitions. Small regions of interest (ROIs), ranging from 4 to 25 pixels, were then chosen within gray and white matter regions, as indicated in the inversion recovery images. These regions were fitted using the gradient echo (38) in the form of the logarithm of the dephasing, namely,

$$
\ln \left[\frac{A(t)}{A_{0}}\right]=\frac{2 \gamma^{2} G^{2} D t^{2+\alpha}}{(2+\alpha) \Gamma(1+\alpha)} .
$$

The fitting was performed using the Levenberg-Marquardt algorithm as implemented in IDL to provide values for $D, \alpha$, and $A_{0}$. This algorithm minimizes the cost function

$$
C=\sum_{i=1}^{n}\left[y_{i}-f\left(\vec{x}_{i}, \vec{a}\right)\right]^{2},
$$

where $y_{i}$ is the experimental diffusion data of length $n$ and $f\left(\vec{x}_{i}, \vec{a}\right)$ is the fitting function to evaluate the variables $\vec{a}$ $=\left[D, \alpha, A_{0}\right]$. The $A_{0}$ term was determined in order to further validate the accuracy of the fit of the equation to the data. When the model is accurate, the image created of each of the $A_{0}$ values, as calculated for each pixel, should represent a copy of the image acquired with no diffusion weighting.

We found the fractional model to fit the experimental data very accurately, with chi-squared values for goodness of fit of $<1 \times 10^{-5}$. Figures $1-3$ show decay curves of the image signal in a region chosen in (a) gray matter and (b) white 

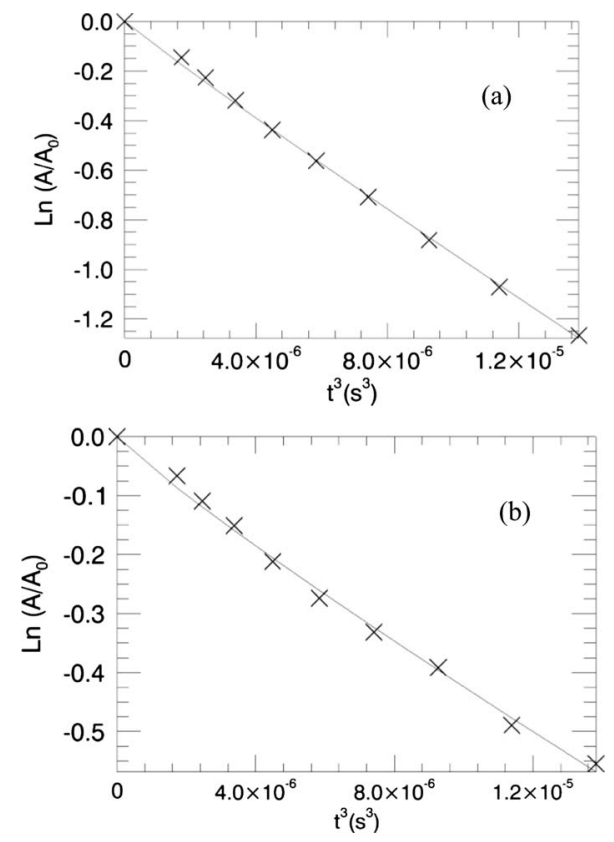

FIG. 3. Plots of data (symbol) and fit (solid line) for (a) gray matter and (b) white matter regions of interest for subject 3 .

matter tissues of three of three subjects. The graphs illustrate fits of Eq. (44) to ROIs drawn in the gray and white matters of three subjects. The values of the fitting parameters $D$ and $\alpha$ are listed in Table I. The image intensity has been normalized by division with the $b_{0}$ image. The curves are plotted versus the time parameter $t^{3}$, with a fixed parameter $G$. Plotting the data in this way shows the nonlinearity of the decay with respect to cubic time and illustrates why the single exponential (3) does not accurately describe the decay. The complexity of the tissue structure causes the water diffusion to change from its free behavior where $\alpha=1$. The diffusion in these experiments exhibited subdiffusive behavior characterized by the fractional parameter $\alpha<1$ with $\alpha=0.28-0.75$. Table II illustrates average values produced for $D$ and $\alpha$ for all subjects in the study. The results from the gradient strength varying experiment are shown in Fig. 4. This experiment demonstrates that, for the more typical diffusionweighted sequence where the degree of diffusion weighting is varied by changing the strength of the applied gradients, the fractional time equation also fits. For this experiment $D$ $=0.52 \times 10^{-3} \mathrm{~mm}^{2} \mathrm{~s}^{-1}$ and $\alpha=0.31$. Maps of the diffusion coefficient $D$, the normalization constant $A_{0}$, and the fractal dimension parameter $\alpha$ were obtained for each voxel in the

TABLE I. Values of $D$ and $\alpha$ for Figs. $1-3$ as obtained using the Levenberg-Marquardt algorithm to fit Eq. (44).

\begin{tabular}{cccccc}
\hline \hline & \multicolumn{2}{c}{ Gray matter } & & \multicolumn{2}{c}{ White matter } \\
\cline { 2 - 3 } \cline { 5 - 6 } Subject & $\begin{array}{c}D \times 10^{-3} \\
\left(\mathrm{~mm}^{2} \mathrm{~s}^{-1}\right)\end{array}$ & $\alpha$ & & $\begin{array}{c}D \times 10^{-3} \\
\left(\mathrm{~mm}^{2} \mathrm{~s}^{-1}\right)\end{array}$ & $\alpha$ \\
\hline 1 & 0.34 & 0.81 & & 0.16 & 0.69 \\
2 & 0.4 & 0.87 & & 0.11 & 0.42 \\
3 & 0.38 & 0.72 & & 0.25 & 0.66 \\
\hline \hline
\end{tabular}

TABLE II. Mean values of $D$ and $\alpha$ with their standard deviations.

\begin{tabular}{lcc}
\hline \hline & $\begin{array}{c}D \times 10^{-3} \\
\left(\mathrm{~mm}^{2} \mathrm{~s}^{-1}\right)\end{array}$ & $\alpha$ \\
\hline Gray matter & $0.48 \pm 0.15$ & $0.77 \pm 0.12$ \\
White matter & $0.19 \pm 0.08$ & $0.5 \pm 19$ \\
\hline \hline
\end{tabular}

images. Figure 5 illustrates some examples of these maps for a selected anatomical slice.

\section{CONCLUDING REMARKS}

In this paper we have shown how the magnetization dephasing in magnetic resonance imaging arising from the Brownian motion of the nuclei in a reservoir of infinite extent may be determined by simply writing the Langevin equation for the phase random variable and then calculating its characteristic function. The method yields in transparent fashion, from the properties of the characteristic function of Gaussian random variables, the classical dephasing results of Carr and Purcell [9], Torrey [15], and Stejskal and Tanner [18] for normal diffusion, which are based on the diffusion limit of the discrete-time random walk proposed by Einstein [10]. Moreover, it is easily generalized to include the inertia of the nuclei, in which the underlying statistics are governed by the Ornstein-Uhlenbeck process [40] and to other more complicated situations where the nuclei move in a field of force of potential $V(\mathbf{r})$. Hence, we have a microscopic explanation of the dephasing process in free water, namely, it results from the nucleus behaving as a random walker executing a jump of finite mean-square length at uniform time intervals, so that the only variable is the orientation of the walker.

The method may also be extended to anomalous diffusion in a transparent fashion in order to provide a possible microscopic justification for the use of stretched exponentials to describe the dephasing in tissue. Namely, the anomalous diffusion may ultimately have its origin in memory effects giving rise to fractional Brownian motion [48]. This process

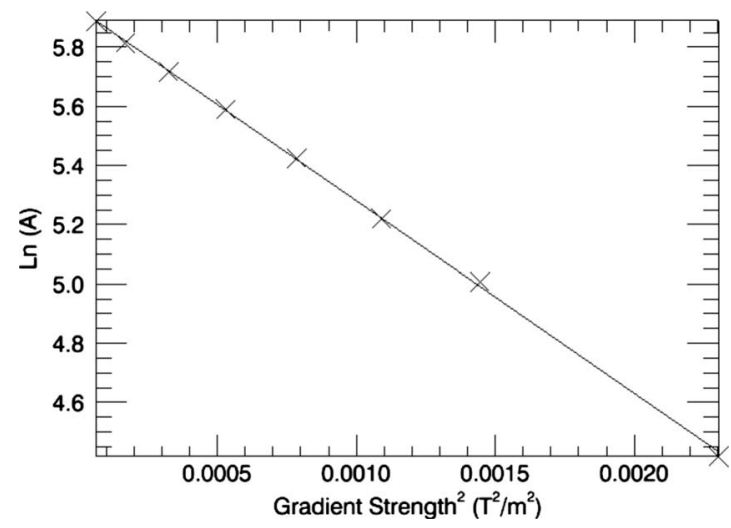

FIG. 4. The data plotted against diffusion-weighting gradient strength squared, with the fit overlaid as a solid line. The region of interest for this plot is chosen from gray matter. 


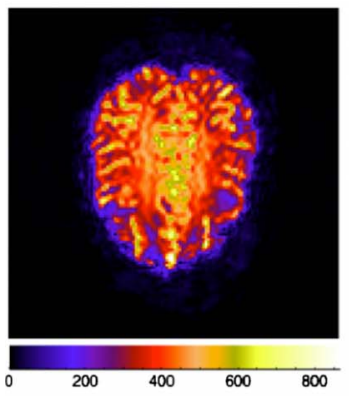

(a)

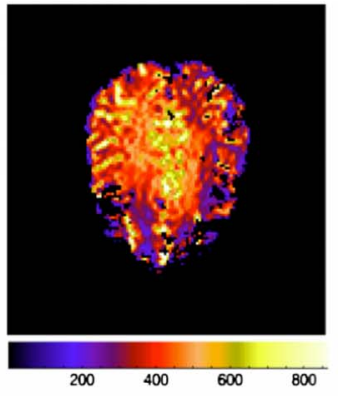

(b)

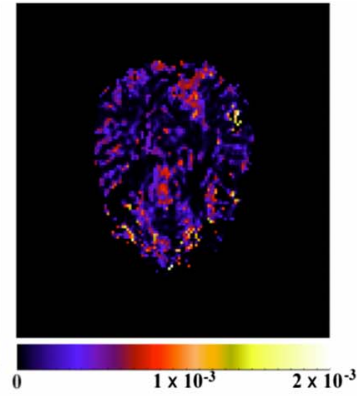

(c)

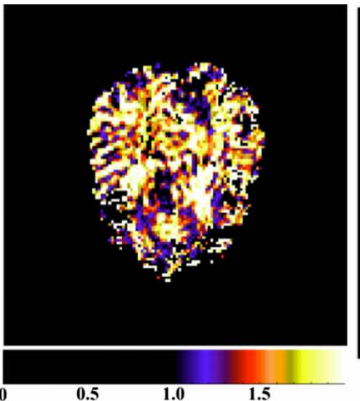

(d)

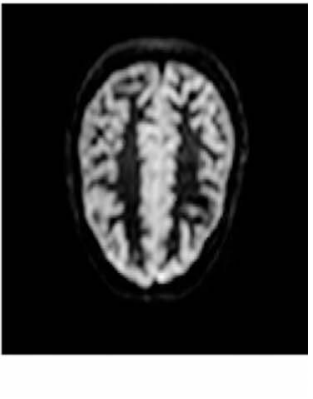

(e)

FIG. 5. (Color online) These images illustrate the success of the fit of the fractional model to the experimental in vivo data, for each voxel in a selected slice. (a) The image acquired with no diffusion weighting, or the $b_{0}$ image. (b)-(d) show maps of the fitting parameters $A_{0}, D$, and $\alpha$ respectively, for each voxel in a given anatomical slice. (b) The representation of each $A_{0}$ produced during a fit of each voxel is the predicted $b_{0}$ image from the fit. (e) This image is an example of an inversion recovery image, which was used to select the regions of interest in gray and white matters for fitting.

naturally introduces the fitting parameter $\alpha$, indicating the role played by fractional dynamics in the time for the complex diffusion, which is observed in human neuronal tissue. In normal diffusion, $\alpha=1$, and we have the classical expressions $[9,15,18]$ once more.

The calculation is accomplished using the fractional Langevin equation for the translational Brownian motion proposed by Lutz [33] which supposes that the random force in that equation is Gaussian, so that the properties of characteristic functions of Gaussian random variables may once again be used to calculate the dephasing yielding a fractional generalization of the results of Refs. $[9,15,18]$. Finally, we may conclude that the fractional diffusion dephasing (44) fits experimental data from gray and white matter accurately and in this complex environment the time dependence of the signal decay is not cubic but falls in the range of $t^{2.1} \rightarrow t^{2.8}$. Such a behavior indicates that one may be observing subdiffusion. This conclusion must always be tempered, however, by the fact that other relaxation mechanisms ultimately resulting from restricted normal diffusion may give rise to seemingly anomalous behavior [17].

\section{ACKNOWLEDGMENTS}

Y.P.K acknowledges financial support from the Royal Irish Academy Mobility Grant 2009. J.M.C. would like to acknowledge financial support from Bruker Biospin, Etlingen, Germany.

\section{APPENDIX A: DIFFUSION EQUATION FOR THE PHASE}

The diffusion equation for the evolution of the probability density function $P\left(x, t \mid x_{0}, t_{0}\right)$ of the random variable $x$ representing the stochastic displacement $X(t)$ in Einstein's theory of the Brownian motion is

$$
\frac{\partial P}{\partial t}=D \frac{\partial^{2} P}{\partial x^{2}} .
$$

By means of the transformation (1) and the chain rule the corresponding diffusion equation for the distribution function $f(\phi, t)$ of the phase $\phi$ is

$$
\frac{\partial f}{\partial t}=D_{\Phi} \frac{\partial^{2} f}{\partial \phi^{2}}
$$

where $\phi$ represents the stochastic phase variable $\Phi(t)$ and we suppose that $\Delta \Phi$ and $\Delta t$ approach zero (extremely small displacements in infinitesimally short times) in such a way that [11]

$$
D_{\Phi}=\lim _{\Delta t \rightarrow 0} \frac{\left\langle\Delta \Phi^{2}\right\rangle}{2 \Delta t}
$$

The diffusion coefficient $D_{\Phi}$ in Eq. (A3) is obtained as follows. The change in the phase $\Phi$ is defined by the Langevin equation (19), so that

$$
\Phi(t)=-\gamma \zeta^{-1} \int_{0}^{t} \lambda\left(t_{1}\right) \int_{0}^{t_{1}} G\left(t^{\prime}\right) d t^{\prime} d t_{1}
$$

assuming that $\Phi(0)=0$. In general, taking account of inertia we would have

$$
\Phi(t)=-\gamma \int_{0}^{t} \dot{X}\left(t_{1}\right) \int_{0}^{t_{1}} G\left(t^{\prime}\right) d t^{\prime} d t_{1}
$$

In order to see how Eq. (A4) allows one to evaluate the diffusion coefficient $D_{\Phi}$ from Eq. (A3), we consider the change $\Delta \Phi^{2}(t)$ in a small time $\Delta t$. According to Eqs. (19), (20), and (A4), we have

$$
\begin{aligned}
\Delta \Phi^{2}(t) & \approx 2 \Phi(t) \dot{\Phi}(t) \Delta t \\
& =\frac{2 \gamma^{2}}{\zeta^{2}} \int_{0}^{t} \int_{0}^{t_{1}} G\left(t^{\prime}\right) d t^{\prime} \int_{0}^{t} G\left(t^{\prime \prime}\right) d t^{\prime \prime} \lambda\left(t_{1}\right) \lambda(t) d t_{1} \Delta t .
\end{aligned}
$$

On taking expectation values, remembering that $G(t)$ is not a stochastic variable and using the properties of the white noise force $\lambda(t)$, we obtain, 


$$
\begin{aligned}
\left\langle\Delta \Phi^{2}\right\rangle & =\frac{4 \zeta k T \gamma^{2}}{\zeta^{2}} \int_{0}^{t} \int_{0}^{t_{1}} G\left(t^{\prime}\right) d t^{\prime} \int_{0}^{t} G\left(t^{\prime \prime}\right) d t^{\prime \prime} \delta\left(t-t_{1}\right) d t_{1} \Delta t \\
& =2 D \gamma^{2}\left(\int_{0}^{t} G\left(t^{\prime}\right) d t^{\prime}\right)^{2} \Delta t .
\end{aligned}
$$

Hence, the desired result is

$$
D_{\Phi}=\left\langle\Delta \Phi^{2}\right\rangle /(2 \Delta t)=D \gamma^{2}\left(\int_{0}^{t} G\left(t^{\prime}\right) d t^{\prime}\right)^{2} .
$$

\section{APPENDIX B: SPIN-ECHO CALCULATION}

The most significant difference between the spin and simple bipolar pulse sequences is the timing gap between the first and second rectangular gradients. We follow the Stejskal and Tanner method [18] and with the notation of Eqs. (2)-(4),

$$
\frac{d A}{d t}=-\gamma^{2} D[F(t) \theta(t)-2 \theta(t-\tau) F(\tau)]^{2} A,
$$

where $F(t)=\int_{0}^{t} G\left(t^{\prime}\right) d t^{\prime}$ and $\theta(t)$ is the unit step function. The $180^{\circ}$ refocusing pulse is placed midway between the two gradient pulses at $t=\tau$. Each of the gradient functions has duration $\tau$ and the second pulse begins at $t=\Delta$. The solution of Eq. (B1) is

$$
A(t)=A(0) \exp \left[-\int_{0}^{t} g\left(t^{\prime}\right) d t^{\prime}\right],
$$

where

$$
\begin{aligned}
\int_{0}^{\tau^{\prime}} g\left(t^{\prime}\right) d t^{\prime}= & -\gamma^{2} D\left[\int_{0}^{\tau^{\prime}} F^{2}\left(t^{\prime}\right) d t^{\prime}-4 F(\tau) \int_{0}^{\tau^{\prime}} F\left(t^{\prime}\right) d t^{\prime}\right. \\
& \left.+4\left(\tau^{\prime}-\tau\right) F^{2}(\tau)\right]
\end{aligned}
$$

The fractional evaluation becomes more complicated, as we must integrate over two arbitrary time intervals $t_{1}$ and $t_{2}$ and, therefore, must define the two gradient integrals, $F\left(t_{1}\right)$ and $F\left(t_{2}\right)$, viz.,

$$
\begin{aligned}
& F\left(t_{1}\right) \rightarrow F\left(t_{1}\right) \theta\left(t_{1}\right)-2 \theta\left(t_{1}-\tau\right) F(\tau), \\
& F\left(t_{2}\right) \rightarrow F\left(t_{2}\right) \theta\left(t_{2}\right)-2 \theta\left(t_{2}-\tau\right) F(\tau) .
\end{aligned}
$$

Recalling Eq. (42), we have

$$
\left\langle\Delta \Phi^{2}\right\rangle(t)=\frac{2 k T \gamma^{2}}{\beta_{\alpha} m \Gamma(\alpha-1)} \int_{0}^{t} d t_{2} \int_{0}^{t_{2}} \frac{F\left(t_{1}\right) F\left(t_{2}\right)}{\left(t_{2}-t_{1}\right)^{2-\alpha}} d t_{1} .
$$

Here, the product of $F\left(t_{1}\right)$ and $F\left(t_{2}\right)$ can be presented as

$$
\begin{aligned}
F\left(t_{1}\right) F\left(t_{2}\right)= & {\left[F\left(t_{1}\right) \theta\left(t_{1}\right)-2 \theta\left(t_{1}-\tau\right) F(\tau)\right] } \\
& \times\left[F\left(t_{2}\right) \theta\left(t_{2}\right)-2 \theta\left(t_{2}-\tau\right) F(\tau)\right] \\
= & F\left(t_{1}\right) F\left(t_{2}\right) \theta\left(t_{1}\right) \theta\left(t_{2}\right)-2 F(\tau) F\left(t_{2}\right) \theta\left(t_{2}\right) \theta\left(t_{1}-\tau\right)
\end{aligned}
$$

$$
\begin{aligned}
& -2 F\left(t_{1}\right) F(\tau) \theta\left(t_{1}\right) \theta\left(t_{2}-\tau\right)+4 F^{2}(\tau) \\
& \times \theta\left(t_{1}-\tau\right) \theta\left(t_{2}-\tau\right) .
\end{aligned}
$$

The evaluation of integrals in Eq. (B4) can then be performed in piecewise fashion provided we can write $F(t)$ explicitly in four intervals of integration, viz.,

$$
(0, \delta),(\delta, \Delta),(\Delta, \Delta+\delta),(\Delta+\delta, 2 \tau) .
$$

The contribution of each term of Eq. (B5) in Eq. (B4) must be evaluated separately for all intervals of integration.

We take, as an example, the first term only, viz., $F\left(t_{1}\right) F\left(t_{2}\right) \theta\left(t_{1}\right) \theta\left(t_{2}\right)$; other terms can be evaluated likewise. The first interval of integration $(0, \delta)$ in Eq. (B4) falls over the first rectangular gradient pulse and, therefore,

$$
F\left(t_{1}\right) F\left(t_{2}\right) \theta\left(t_{1}\right) \theta\left(t_{2}\right)=G^{2} t_{1} t_{2} \theta\left(t_{1}\right) \theta\left(t_{2}\right) .
$$

Inserting Eq. (B4) into Eq. (B6) and performing the integrations yields

$$
\begin{aligned}
\left.\left\langle\Delta \Phi^{2}\right\rangle(t)\right|_{1(1)} & =\frac{2 k T \gamma^{2}}{m \beta_{\alpha} \Gamma(\alpha-1)} \int_{0}^{\delta} d t_{2} \int_{0}^{t_{2}} \frac{G^{2} t_{1} t_{2}}{\left(t_{2}-t_{1}\right)^{2-\alpha}} d t_{1} \\
& =\frac{2 k T \gamma^{2} G^{2} \delta^{2+\alpha}}{m \beta_{\alpha}(2+\alpha) \Gamma(1+\alpha)} .
\end{aligned}
$$

During the second time interval from $\delta$ to $\Delta, G=0$ and thus $F\left(t_{1}\right)$ and $F\left(t_{2}\right)$ remain constant, so that $F\left(t_{1}\right) F\left(t_{2}\right) \theta\left(t_{1}\right) \theta\left(t_{2}\right)$ $=G^{2} \delta^{2} \theta\left(t_{1}\right) \theta\left(t_{2}\right)$ and

$$
\begin{aligned}
\left.\left\langle\Delta \Phi^{2}\right\rangle(t)\right|_{1(2)} & =\frac{2 k T \gamma^{2}}{m \beta_{\alpha} \Gamma(\alpha-1)} \int_{\delta}^{\Delta} d t_{2} \int_{\delta}^{t_{2}} \frac{G^{2} \delta^{2}}{\left(t_{2}-t_{1}\right)^{2-\alpha}} d t_{1} \\
& =\frac{2 k T \gamma^{2} G^{2} \delta^{2}}{m \beta_{\alpha} \Gamma(1+\alpha)}(\Delta-\delta)^{\alpha} .
\end{aligned}
$$

The third time interval includes the second gradient pulse, from $\Delta$ to $\Delta+\delta$. Here,

$$
\begin{aligned}
F\left(t_{1}\right) F\left(t_{2}\right) \theta\left(t_{1}\right) \theta\left(t_{2}\right)= & \left(G t_{1}-G \Delta+G \delta\right)\left(G t_{2}-G \Delta\right. \\
& +G \delta) \theta\left(t_{1}\right) \theta\left(t_{2}\right)
\end{aligned}
$$

and

$$
\begin{aligned}
\left.\left\langle\Delta \Phi^{2}\right\rangle(t)\right|_{1(3)}= & \frac{2 k T \gamma^{2} G^{2}}{m \beta_{\alpha} \Gamma(\alpha-1)} \int_{\Delta}^{\Delta+\delta} \\
& \times\left(t_{2}-\Delta+\delta\right) d t_{2} \int_{\Delta}^{t_{2}} \frac{\left(t_{1}-\Delta+\delta\right)}{\left(t_{2}-t_{1}\right)^{2-\alpha}} d t_{1} \\
= & \frac{2 k T \gamma^{2} G^{2}(5+2 \alpha)}{m \beta_{\alpha}(2+\alpha) \Gamma(1+\alpha)} \delta^{2+\alpha} .
\end{aligned}
$$

Finally, in the interval from the end of the second gradient at $t=\Delta+\delta$ to $2 \tau, G=0$ and $F\left(t_{1}\right)$ and $F\left(t_{2}\right)$ are constant, so that $F\left(t_{1}\right) F\left(t_{2}\right) \theta\left(t_{1}\right) \theta\left(t_{2}\right)=4 G^{2} \delta^{2} \theta\left(t_{1}\right) \theta\left(t_{2}\right)$ and 


$$
\begin{aligned}
\left.\left\langle\Delta \Phi^{2}\right\rangle(t)\right|_{1(4)} & =\frac{2 k T \gamma^{2}}{m \beta_{\alpha} \Gamma(\alpha-1)} \int_{\Delta+\delta}^{2 \tau} d t_{2} \int_{\Delta+\delta}^{t_{2}} \frac{(2 G \delta)^{2}}{\left(t_{2}-t_{1}\right)^{2-\alpha}} d t_{1} \\
& =\frac{8 k T \gamma^{2} G^{2} \delta^{2}}{m \beta_{\alpha} \Gamma(1+\alpha)}(2 \tau-\delta-\Delta)^{\alpha} .
\end{aligned}
$$

The sum of Eqs. (B7)-(B10) yields the contribution of the first term,

$$
\begin{aligned}
\left.\left\langle\Delta \Phi^{2}\right\rangle(t)\right|_{1}= & \frac{2 k T \gamma^{2} G^{2} \delta^{2}}{m \beta_{\alpha} \Gamma(1+\alpha)} \\
& \times\left\{\frac{2(3+\alpha)}{2+\alpha} \delta^{\alpha}+(\Delta-\delta)^{\alpha}+4(2 \tau-\delta-\Delta)^{\alpha}\right\} .
\end{aligned}
$$

Each of the remaining three terms in Eq. (B7) must be treated similarly, however, only in the range from $\tau$ to $2 \tau$, as they are zero for $t<\tau$. The results are, respectively,

$$
\begin{aligned}
\left.\left\langle\Delta \Phi^{2}\right\rangle(t)\right|_{2}= & \left.\left\langle\Delta \Phi^{2}\right\rangle(t)\right|_{3} \\
= & -\frac{4 k T \gamma^{2} G^{2} \delta^{2}}{m \beta_{\alpha} \Gamma(1+\alpha)} \\
& \times\left[\frac{(1+2 \alpha) \delta^{\alpha}}{1+\alpha}+(\Delta-\tau)^{\alpha}+2(2 \tau-\Delta-\delta)^{\alpha}\right], \\
\left.\left\langle\Delta \Phi^{2}\right\rangle(t)\right|_{4}= & \frac{8 k T \gamma^{2} G^{2} \delta^{2}}{m \beta_{\alpha} \Gamma(1+\alpha)}\left[\delta^{\alpha}+(\Delta-\tau)^{\alpha}+(2 \tau-\Delta-\delta)^{\alpha}\right] .
\end{aligned}
$$

When all of them have been evaluated and summed, the resulting equation for the fractional diffusion spin-echo experiment is given by Eq. (43).
[1] M. E. Moseley, J. Kucharczyk, J. Mintorovitch, Y. Cohen, J. Kurhanewicz, N. Derugin, H. Asgari, and D. Norman, AJNR 11, 423 (1990).

[2] A. Darquie, J. B. Poline, C. Poupon, H. Saint-James, and D. Le Bihan, Proc. Natl. Acad. Sci. U.S.A. 98, 9391 (2001).

[3] P. J. Basser, J. Mattiello, and D. Le Bihan, Biophys. J. 66, 259 (1994).

[4] R. Bammer and F. Fazekas, Neuroimaging Clin. N. Am. 12, 71 (2002).

[5] M. Maeda, Y. Kawamura, Y. Tamagawa, T. Matsuda, S. Itoh, H. Kimura, T. Iwasaki, N. Hayashi, K. Yamamoto, and Y. Ishii, J. Comput. Assist. Tomogr. 28, 540 (2004).

[6] F. Bloch, Phys. Rev. 70, 460 (1946).

[7] E. L. Hahn, Phys. Rev. 80, 580 (1950).

[8] T. P. Das and A. K. Saha, Phys. Rev. 93, 749 (1954).

[9] H. Y. Carr and E. M. Purcell, Phys. Rev. 94, 630 (1954).

[10] A. Einstein, in Investigations on the Theory of the Brownian Movement, edited with notes by R. Fuerth (Methuen, London, 1926); Investigations on the Theory of the Brownian Movement, reprinted ed. (Dover, New York, 1954); M. D. Haw, J. Phys.: Condens. Matter 14, 7769 (2002).

[11] W. T. Coffey, Yu. P. Kalmykov, and J. T. Waldron, The Langevin Equation, 2nd ed. (World Scientific, Singapore, 2004).

[12] W. Coffey, Adv. Chem. Phys. 63, 69 (1985).

[13] M. Kac, Am. Math. Monthly 54, 369 (1947); reprinted in Selected Papers on Noise and Stochastic Processes, edited by N. Wax (Dover, New York, 1954).

[14] M. C. Wang and G. E. Uhlenbeck, Rev. Mod. Phys. 17, 323 (1945).

[15] H. C. Torrey, Phys. Rev. 104, 563 (1956).

[16] A. Abragam, The Principles of Nuclear Magnetism (Oxford University Press, London, 1961).

[17] D. S. Grebenkov, Rev. Mod. Phys. 79, 1077 (2007).

[18] E. O. Stejskal and J. E. Tanner, J. Chem. Phys. 42, 288 (1965).

[19] T. Niendorf, D. G. Norris, and D. Leibfritz, Magn. Reson. Med. 32, 672 (1994).

[20] T. Q. Duong, J. J. H. Ackerman, H. S. Ying, and J. J. Neil, Magn. Reson. Med. 40, 1 (1998); T. Q. Duong, J. V. Sehy, D. A. Yablonskiy, B. J. Snider, J. J. H. Ackerman, and J. J. Neil, ibid. 45, 801 (2001).

[21] K. M. Bennett, J. S. Hyde, and K. M. Schmainda, Magn. Reson. Med. 56, 235 (2006).

[22] W. T. Coffey, J. Mol. Liq. 114, 5 (2004).

[23] Fractals, Diffusion and Relaxation in Disordered Complex Systems, Advances in Chemical Physics, edited by W. T. Coffey and Yu. P. Kalmykov (Wiley, New York, 2006), Vol. 133, parts A \& B.

[24] S. I. Denisov, P. Hänggi, and H. Kantz, EPL 85, 40007 (2009).

[25] W. T. Coffey, D. S. F. Crothers, D. Holland, and S. V. Titov, J. Mol. Liq. 114, 165 (2004).

[26] E. Lutz, Phys. Rev. Lett. 86, 2208 (2001).

[27] G. A. Losa, D. Merlini, T. F. Nonnenmacher, and E. R. Weibel, Fractals in Biology and Medicine (Birkhauser, Basel, 1997), Vol. II.

[28] I. Goychuk, E. Heinsalu, M. Patriarca, G. Schmidt, and P. Hänggi, Phys. Rev. E 73, 020101(R) (2006).

[29] R. Metzler and J. Klafter, Phys. Rep. 339, 1 (2000).

[30] I. M. Sokolov, J. Klafter, and A. Blumen, Phys. Today 55(11), 48 (2002).

[31] H. Yang, G. B. Luo, P. Karnchanaphanurach, T. M. Louie, I. Rech, S. Cova, L. Y. Xun, and X. S. Xie, Science 302, 262 (2003); S. C. Kou and X. S. Xie, Phys. Rev. Lett. 93, 180603 (2004).

[32] A. Widom and H. J. Chen, J. Phys. A 28, 1243 (1995).

[33] E. Lutz, Phys. Rev. E 64, 051106 (2001).

[34] R. L. Magin, O. Abdullah, D. Baleanu, and X. J. Zhou, J. Magn. Reson. 190, 255 (2008); R. L. Magin, X. Feng, and D. Baleanu, Concepts Magn. Reson., Part A 34, 16 (2009).

[35] B. Robertson, Phys. Rev. 151, 273 (1966).

[36] E. O. Stejskal and J. E. Tanner, J. Chem. Phys. 49, 1768 (1968).

[37] P. T. Callaghan, A. Coy, D. McGowan, K. J. Packer, and F. O. Zelaya,Nature (London) 351, 467 (1991).

[38] S. D. Stoller, W. Happer, and F. J. Dyson, Phys. Rev. A 44, 7459 (1991).

[39] P. N. Sen, Concepts Magn. Reson. 23A, 1 (2004).

[40] G. E. Uhlenbeck and L. S. Ornstein, Phys. Rev. 36, 823 (1930). 
[41] J. Stepišnik, Prog. Nucl. Magn. Reson. Spectrosc. 17, 187 (1985).

[42] J. Stepišnik and P. T. Callaghan, Physica B 292, 296 (2000).

[43] J. Stepišnik, Physica B 183, 343 (1993).

[44] B. J. West, M. Bologna, and P. Grigolini, Physics of Fractal Operators (Springer-Verlag, New York, 2003).

[45] K. B. Oldham and J. Spanier, The Fractional Calculus (Aca- demic Press, New York, 1974).

[46] W. R. Schneider and W. Wyss, J. Math. Phys. 30, 134 (1989).

[47] L. L. Latour, K. S. Svoboda, P. P. Mitra, and C. H. Sotak, Proc. Natl. Acad. Sci. U.S.A. 91, 1229 (1994).

[48] B. B. Mandelbrot and J. W. Van Ness,SIAM Rev. 10, 422 (1968). 